\title{
Distribution of lanthanum carbonate in the gastric mucosa confirmed by electron microscopy with a magnified endoscopy: a case report and literature review
}

\author{
Takahiro Yamada ${ }^{1} \cdot$ Tsuneo Oyama $^{2} \cdot$ Akihisa Tomori $^{1} \cdot$ Akiko Takahashi $^{2} \cdot$ Kiyokazu Kametani $^{3} \cdot$ Satoshi Shiozawa $^{4}$. \\ Hiroyoshi Ota ${ }^{5} \cdot$ Tadakazu Shimoda $^{6}$
}

Received: 28 June 2019 / Accepted: 20 November 2019 / Published online: 3 December 2019

(c) The Author(s) 2019

\begin{abstract}
We describe the case of a 70-year-old man with diabetic nephropathy undergoing hemodialysis. Four years following hemodialysis, he started taking lanthanum carbonate $1500 \mathrm{mg} /$ day and lansoprazole $30 \mathrm{mg} /$ day. Nine years following hemodialysis, he underwent screening esophagogastroduodenoscopy, which demonstrated the presence of the whitish cobblestone-like mucosa in the gastric corpus and multiple reddish depressed lesions with annular whitish mucosa in the antrum. With magnified narrow-band imaging endoscopy, a yellowish-white substance was observed in the villous structure, and subepithelial vessels were observed on the yellowish-white substance. Biopsies were taken from the whitish cobblestone-like mucosa of the upper corpus, a reddish depressed part of the antrum. Histologically, aggregates of cells containing amphophilic fine granular material were found in the mucosal interstitium. These cells stained positive for CD68 and were identified as histiocytes. Since he had been taking lanthanum carbonate for 5 years, we considered the possibility of histiocyte-mediated phagocytosis of lanthanum. Digital mapping via scanning electron microscopy with energy-dispersive X-ray spectrometry showed the presence of lanthanum and phosphorus in the interstitium and cytoplasm of histiocytes. The white, rough mucosa in the gastric body appeared 6 months following the commencement of lanthanum administration and still exists 3 years and 5 months after discontinuation of lanthanum.
\end{abstract}

Keywords Gastric mucosa $\cdot$ Lanthanum $\cdot$ Retrospective

Takahiro Yamada

hardswimdrummer@yahoo.co.jp

1 Department of Gastroenterology, Saku Central Hospital Advanced Care Center, 3400-28 Nakagomi, Saku, Nagano 385-0051, Japan

2 Department of Endoscopy, Saku Central Hospital Advanced Care Center, Saku, Nagano, Japan

3 Division of Instrumental Analysis, Research Center for Human and Environmental Sciences, Shinshu University, Matsumoto, Nagano, Japan

4 Clinical Pathology, Saku Central Hospital Advanced Care Center, Saku, Nagano, Japan

5 Department of Biomedical Laboratory Sciences, School of Health Sciences, Shinshu University School of Medicine, Matsumoto, Nagano, Japan

6 Department of Pathology, Shizuoka Cancer Center, Shizuoka, Japan

\section{Introduction}

Lanthanum carbonate is a powerful phosphate-binding agent used to treat hyperphosphatemia in patients with chronic renal failure. It binds with phosphate groups to form a highly insoluble substance. Lanthanum carbonate itself is insoluble, and its rate of absorption via the intestine is reported to be only $0.00127 \%$ [1]. Histopathologically, lanthanum deposition in the gastric mucosa was first demonstrated by Makino et al. in 2015 [2]. To date, numerous case reports have been published on lanthanum deposition [3-17]. Previous reports of endoscopic findings of lanthanum deposition in the gastric mucosa have varied widely. Specifically, Iwamuro et al. reported that gastric mucosa with annular whitish mucosa, diffuse whitish mucosa, and whitish spots visible by endoscopy may be characteristic clinical features of lanthanum deposition [14, 16, 17]. However, no studies have been conducted to address the endoscopic findings of lanthanum deposition in the gastric mucosa using magnified 
narrow-band imaging (NBI) endoscopy in detail. Furthermore, although previous reports demonstrated the existence of lanthanum by scanning electron microscopy (SEM), they did not describe where lanthanum exists.

\section{Case report}

The patient was a 70-year-old man with no particular complaints. He had a previous history of diabetic nephropathy, cerebral infarction, and colorectal cancer. Four years following hemodialysis, he began taking lanthanum carbonate $1500 \mathrm{mg} /$ day and lansoprazole $30 \mathrm{mg} /$ day. Blood test results revealed severe renal impairment during dialysis treatment, and the patient exhibited elevated levels of magnesium, phosphate, and glycated hemoglobin A1c. Serum Helicobacter pylori antibody levels were found to be negative (8.7 IU/ ml) (Table 1).

He underwent screening esophagogastroduodenoscopy (EGD), which revealed whitish cobblestone-like mucosa $[18,19]$ in the gastric corpus (Fig. 1a) and depressed red lesions surrounded by annular whitish mucosa in the antrum (Fig. 1b). With magnified NBI endoscopy, a yellowish-white substance was observed within regular villous-like structures, and a yellowish-white substance was observed above enlarged regular vessels (Fig. 1c, d).

Biopsies were taken from three locations: an area of whitish, rough granular mucosa on the posterior wall of the upper corpus, a red depressed lesion in the greater curvature of the antrum, and annular whitish mucosa surrounding a depressed lesion. Hyperplasia of parietal cells was observed histologically, which was thought to be due

Table 1 Laboratory data

Hematology test results

WBC count $5.6 \times 10^{3} / \mu \mathrm{L}$

RBC count $340 \times 10^{4} / \mu \mathrm{L}$

UN $56 \mathrm{mg} / \mathrm{dL}$

$\mathrm{Hb}$ level $10.7 \mathrm{~g} / \mathrm{dL}$

CRE $14.2 \mathrm{mg} / \mathrm{dL}$

PLT count $12.0 \times 10^{4} / \mu \mathrm{L}$

$\mathrm{Na} 140 \mathrm{mmol} / \mathrm{L}$

$\mathrm{K} 5.6 \mathrm{mmol} / \mathrm{L}$

TP level $6.8 \mathrm{~g} / \mathrm{dL}$

$\mathrm{Cl} 107 \mathrm{mmol} / \mathrm{L}$

Ca $9.1 \mathrm{mg} / \mathrm{dL}$

P $6.6 \mathrm{mg} / \mathrm{dL}$

$\mathrm{Mg} 2.9 \mathrm{mg} / \mathrm{dL}$

GLU $149 \mathrm{mg} / \mathrm{dL}$

$\mathrm{HbA1c} 6.6 \%$

LDH level $156 \mathrm{U} / \mathrm{L}$

Anti-Helicobacter pylori immunoglobulin $\mathrm{G}$ antibodies $8.7 \mathrm{U} / \mathrm{mL}$

$W B C$ white blood cell, $R B C$ red blood cell, $H b$ hemoglobin, PLT platelet, $T P$ total protein, $A L B$ albumin, $A S T$ aspartate aminotransferase, $A L T$ alanine aminotransferase, $A L P$ alkaline phosphatase, $L D H$ lactate dehydrogenase, $C R E$ creatinine, $N a$ sodium, $K$ potassium, $\mathrm{Cl}$ chloride, $\mathrm{Ca}$ calcium, $\mathrm{P}$ phosphorous, $\mathrm{Mg}$ magnesium, $G L U$ glucose, $H b A l c$ glycated hemoglobin A1c to the lansoprazole ingestion, resulting in the cobblestonelike appearance of the mucosa. And aggregates of cells containing amphophilic fine granular material together with coarser brown to deep purple material were observed in the mucosal interstitium of the lamina propria at all biopsy sites by hematoxylin-eosin staining (Fig. 2a). These cells stained positive for CD68 and were identified as histiocytes (Fig. 2b). Considering that the patient had been taking lanthanum carbonate, it was hypothesized that the histiocytes might have phagocytosed the heavy metal lanthanum. Thus, we decided to perform SEM-EDS for the element analysis of the deposited materials.

Spectral analysis by EDS characterized the constituent elements of the samples, and deposits of lanthanum and phosphorus were detected. A change in color, observed during the element analysis performed by digital mapping via SEM-EDS, indicated a change in element concentrations. Green and red indicated the presence of lanthanum and phosphorus, respectively, and brown spots formed in the presence of a lanthanum and phosphorus complex. Both lanthanum and phosphorus were primarily found in histiocytes, with partial deposition in the interstitium (Fig. 3a-c).

Subsequently, the patient stopped taking lanthanum, and we continued to perform EGD regularly. Three years and 5 months after discontinuation of lanthanum, whitish rough mucosa and depressed lesions surrounded by annular whitish mucosa improved a little (Fig. 4a, b), and the number of histiocytes has decreased (Fig. 5).

\section{Discussion}

Dialysis patients with chronic kidney disease develop hyperphosphatemia due to their decreased capacity of phosphate excretion. Since hyperphosphatemia causes secondary hyperparathyroidism, it used to be formerly treated with aluminum agents; however, these caused problematic side effects including encephalopathy. In 2009, lanthanum carbonate was approved for coverage by Japanese health insurance. Lanthanum is assumed to be excreted from the body by secretion into bile [1]. It is known that absorbed lanthanum is deposited in the liver and bone, but since the amounts are miniscule, it had been thought not to cause organ damage. However, histopathological deposition of lanthanum has been demonstrated in the gastric mucosa [2].

Some previous reports have described various characteristics, including elevations, erosion, and ulceration [3-10, 12-15], whereas others have described the presence of whitish, rough granular mucosa [2,11, 14-17]. Table 2 summarizes 51 cases in 13 studies that clearly describe the endoscopic findings of lanthanum deposition in the gastric mucosa, and diagnose it by biopsy. Whitish mucosa was evident in $70.6 \%$ of these cases (36/51). 

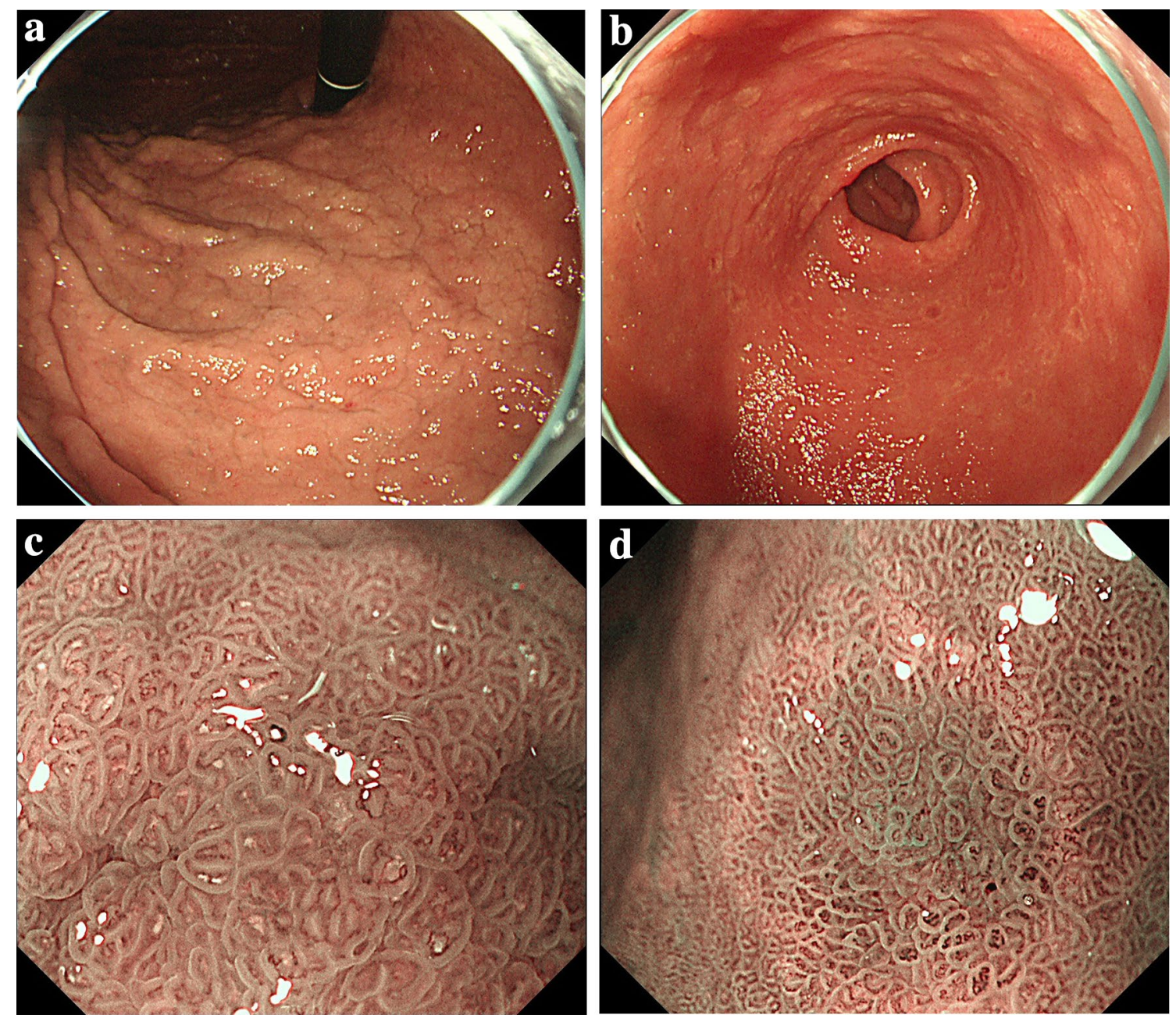

Fig. 1 Upper gastrointestinal endoscopic findings. a Whitish, rough mucosa is present in the gastric corpus. b Depressed red lesions are surrounded by annular yellowish mucosa in the antrum. c, d With magnified NBI endoscopy, a yellowish-white substance was observed within regular villous-like structures. And a yellowish-white substance was observed above enlarged regular vessels

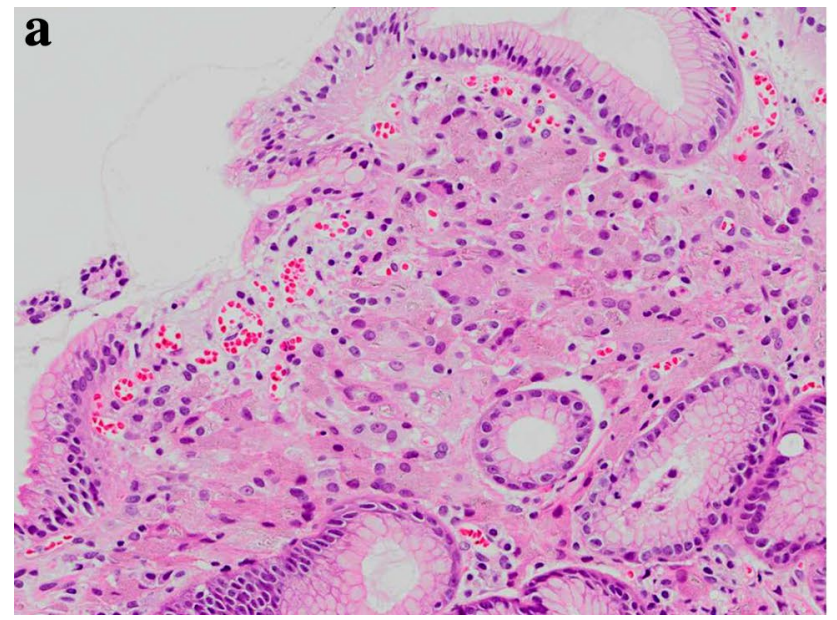

Fig. 2 Examination of biopsy tissue specimens. a, b Aggregates of cells containing amphophilic fine granular material together with coarser brown to deep purple material were observed in the mucosal

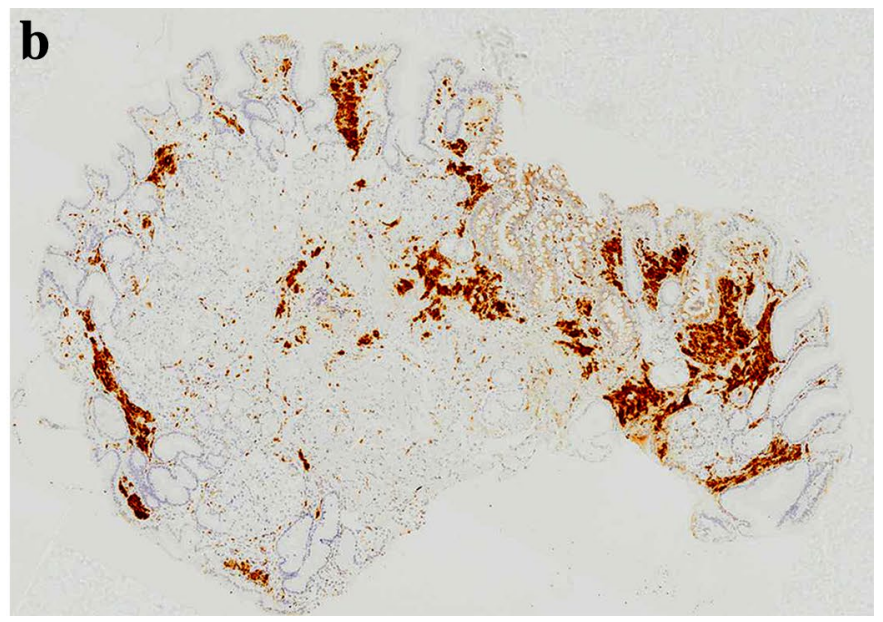

interstitium of the lamina propria at all biopsy sites by hematoxylineosin staining and these cells stained positive for CD68 
Fig. 3 Scanning electron microscopic findings. Green (a), red (b), and brown spots (c) indicate the presence of lanthanum, phosphorus, and a complex of lanthanum and phosphorus, respectively. There is a histiocyte in the center of the figure. Lanthanum, phosphorus, and the complexes are mainly present in histiocytes and partially present in the interstitium
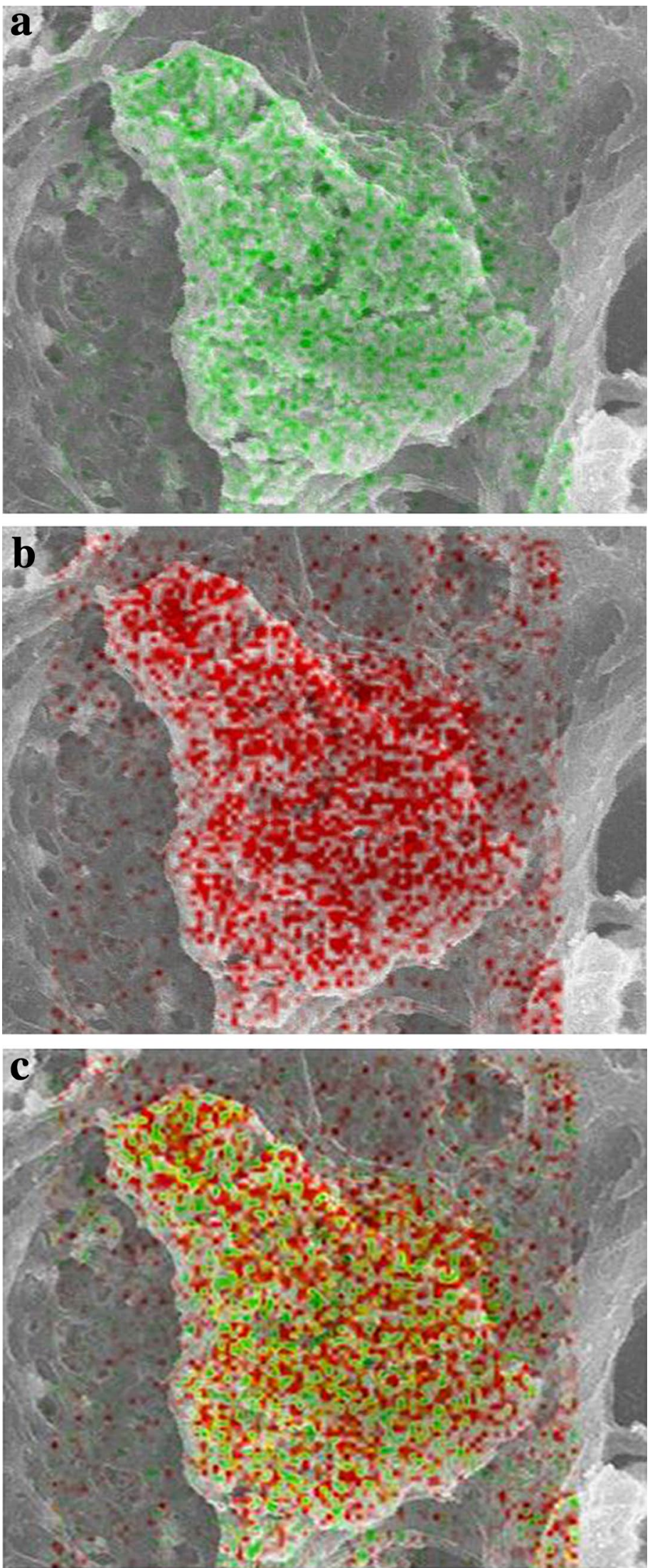

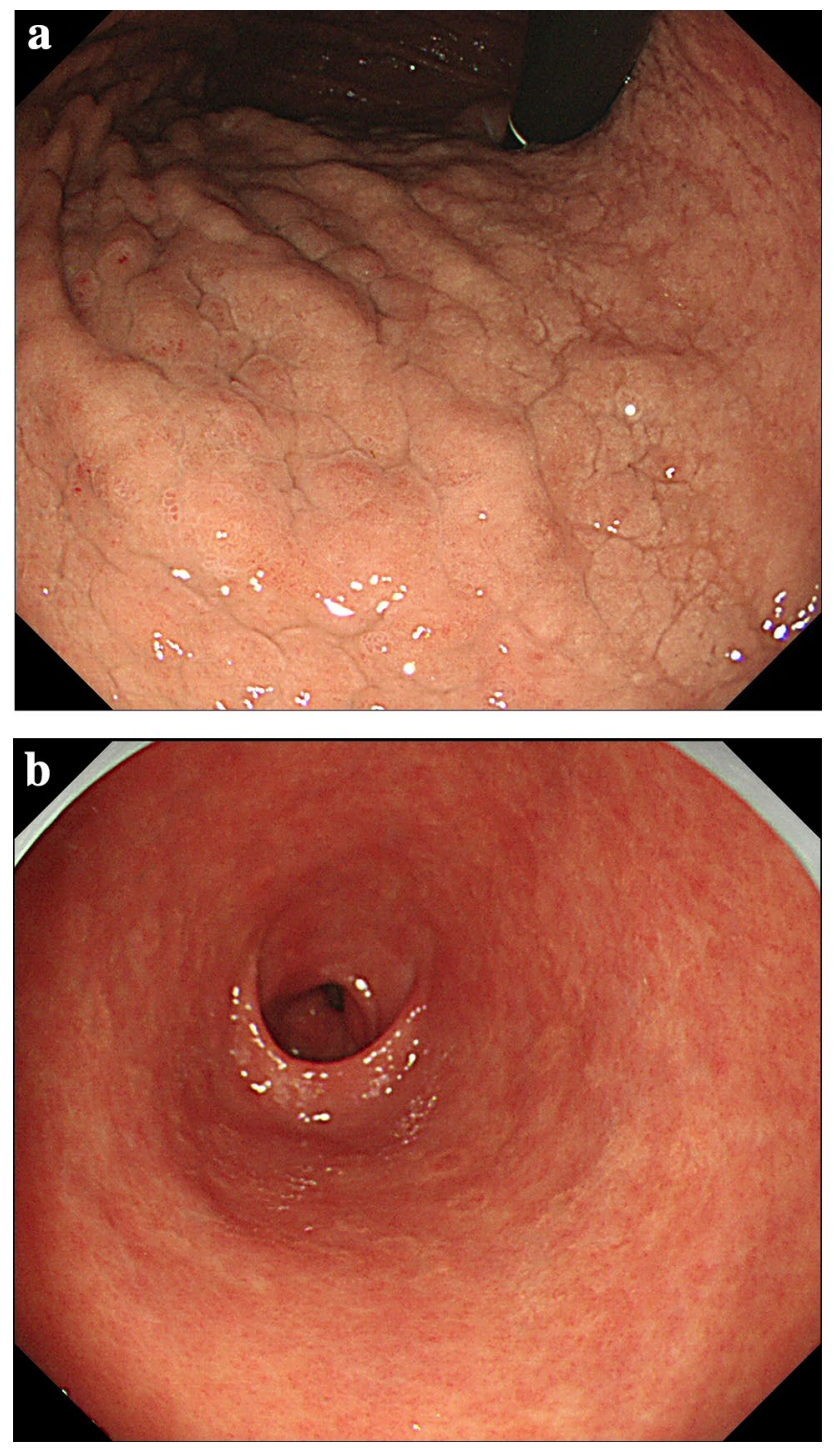

Fig. 4 Follow-up upper gastrointestinal endoscopic findings. a, b Esophagogastroduodenoscopy at 3 years and 5 months after lanthanum discontinuation. The whitish, rough mucosa and depressed lesions improved

The time from the start of lanthanum administration to histological diagnosis varied from 3 to 60 months (mean, 34.5 months). The author suggested that histologically, at least 3 months may be required for the occurrence of lanthanum phagocytosis after the start of lanthanum treatment. In previously reported cases within 12 months starting lanthanum administration with detailed endoscopic findings, whitish mucosa was evident in $83.3 \%(5 / 6)$ of cases in the early stage. This finding suggests that it may be an important indicator of possible lanthanum deposition in the gastric mucosa. Our retrospective review of EGD findings in the present case showed that the whitish cobblestone-like mucosa in the gastric body appeared 6 months after the start of lanthanum administration (Fig. 6).

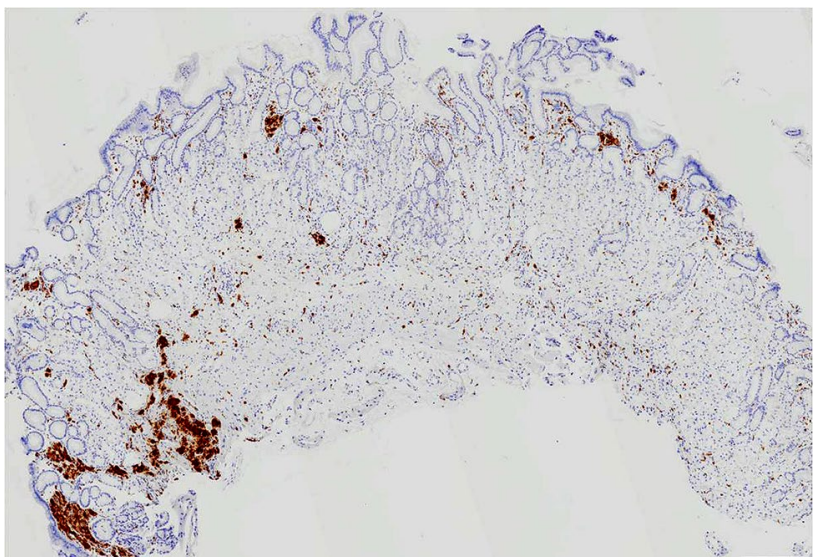

Fig. 5 Histology of follow-up biopsy. Number of histiocytes decreased

Table 2 Previously reported endoscopic findings in patients with lanthanum deposition in the gastric mucosa

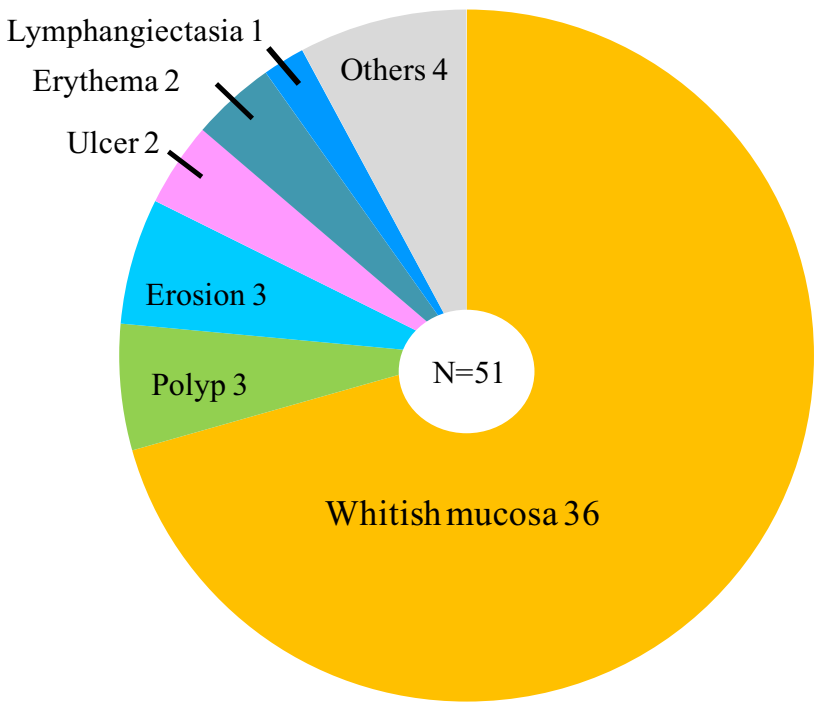

No studies have been conducted to address the endoscopic findings of lanthanum deposition in the gastric mucosa using magnified NBI endoscopy in detail. In our patient, magnified NBI endoscopy revealed regular villous structures containing a yellowish-white substance; since this was detected below the epithelial vessels, it indicated the presence of a substance in the interstitium of the lamina propria. Based on the result of pathological tests and SEM, we considered that lanthanum had been taken up by histiocytes.

It is important to distinguish between the yellowish-white substance seen by the magnified NBI endoscopy and "white opaque substance" (WOS) [20, 21]. Yao et al. reported WOS obscures the microvessels that are just beneath the epithelium. However, in the present case, we could see microvessels on the yellowish-white substance, meaning that the yellowish-white substance exists beneath the sub-epithelial 


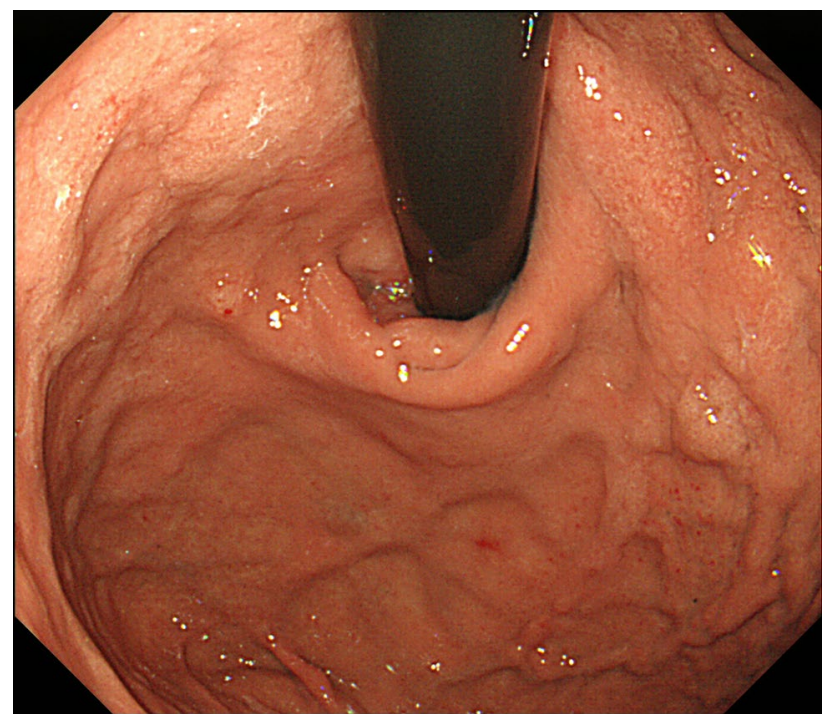

Fig. 6 Retrospective review of upper gastrointestinal endoscopy. Esophagogastroduodenoscopy performed 5 years prior to performing the first biopsy shows a whitish cobblestone-like mucosa in the gastric body. Lanthanum was started 6 months prior

capillary network. Therefore, it can be concluded that magnified NBI endoscopy can be helpful in distinguishing between the uptake of lanthanum and WOS.

Our patient stopped taking lanthanum carbonate after its deposition was confirmed in the gastric mucosa. However, there have been no significant changes in the endoscopic findings, with the persistence of whitish granular mucosa in the gastric corpus and the depressed lesions surrounded by annular whitish mucosa in the antrum. As needed, we will continue to monitor his condition closely to observe any further changes.

In the present case, lanthanum deposition in the gastric mucosa was demonstrated by SEM-EDS. In SEM, an electron beam is directed at the sample, and electrons and X-rays discharged by the sample are detected to provide a threedimensional picture of its surface structure. An energy-dispersive X-ray spectroscope is a device attached to a scanning electron microscope that detects characteristic X-rays and obtains information on the elements present in a given sample. "Characteristic X-rays" are multiple X-rays of varying energies characteristic of a particular element, and these are emitted when electrons from an electron beam collide with the atoms in a substance. Since each element emits a different pattern of characteristic X-rays, measuring the energy of the characteristics X-rays enables us to identify the elements present in the area irradiated by the electron beam. There are two methods used for EDS analysis: (1) spectral analysis to determine the elements deposited in the sample, (2) digital mapping for investigating the location of the deposited elements.
In our patient, histiocytes were identified by SEM, and digital mapping by EDS demonstrated the dense deposition of lanthanum phosphate in histiocytes. Lanthanum phosphate was also demonstrated in the interstitium, showing that this agent was not only taken up by histiocytes but also deposited in the interstitium. Previous studies have also demonstrated lanthanum phosphate deposition by SEM, but they did not show lanthanum phosphate deposition in the interstitium. Hence, our results provide evidence suggesting that free lanthanum phosphate in the interstitium is phagocytosed by histiocytes, offering an insight into the process of lanthanum phosphate deposition.

In summary, we reported that the case of lanthanum uptake by histiocytes in gastric mucosa has characteristic findings by magnified NBI endoscopy. We observed a yellowish-white substance within regular villous-like structures and enlarged regular vessels by magnified NBI endoscopy. Additionally, we proved that lanthanum together with phosphorus were densely distributed in histiocytes and sparsely in the interstitium by digital mapping via SEM-EDS.

\section{Compliance with ethical standards}

Conflict of interest The authors declare that they have no conflict of interest.

Human rights All procedures followed were in accordance with the ethical standards of the responsible committee on human experimentation (institutional and national) and with the Helsinki Declaration of 1975, as revised in 2008.

Informed consent Informed consent was obtained from the patient to be included in the study.

Open Access This article is distributed under the terms of the Creative Commons Attribution 4.0 International License (http://creativeco mmons.org/licenses/by/4.0/), which permits unrestricted use, distribution, and reproduction in any medium, provided you give appropriate credit to the original author(s) and the source, provide a link to the Creative Commons license, and indicate if changes were made.

\section{References}

1. Pennick M, Dennis K, Damment SJ. Absolute bioavailability and disposition of lanthanum in healthy human subjects administered lanthanum carbonate. J Clin Pharmacol. 2006;46:738-46.

2. Makino M, Kawaguchi K, Shimojo H, et al. Extensive lanthanum deposition in the gastric mucosa: the first histopathological report. Pathol Int. 2015;65:33-7.

3. Tonooka A, Uda S, Tanaka $\mathrm{H}$, et al. Possibility of lanthanum absorption in the stomach. Clin Kidney J. 2015;8:572-5.

4. Rothenberg ME, Araya H, Longacre TA, et al. Lanthanum-induced gastrointestinal histiocytosis. ACG Case Rep J. 2015;2:187-9.

5. Haratake J, Yasunaga C, Ootani A, et al. Peculiar histiocytic lesions with massive lanthanum deposition in dialysis 
patients treated with lanthanum carbonate. Am J Surg Pathol. 2015;39:767-71.

6. Yasunaga C, Haratake J, Ohtani A. Specific accumulation of lanthanum carbonate in the gastric mucosal histiocytes in a dialysis patient. Ther Apher Dial. 2015;19:622-4.

7. Valka AK, Jain D, Jaffe PE, et al. A nodular foreign body reaction in a dialysis patient receiving long-term treatment with lanthanum carbonate. Am J Kidney Dis. 2016;67:128-32.

8. Goto K, Ogawa K. Lanthanum deposition is frequently observed in the gastric mucosa of dialysis patients with lanthanum carbonate therapy: a clinicopathologic study of 13 cases, including 1 case of lanthanum granuloma in the colon and 2 nongranulomatous gastric cases. Int J Surg Pathol. 2016;24:89-92.

9. Yabuki K, Shiba E, Harada H, et al. Lanthanum deposition in the gastrointestinal mucosa and regional lymph nodes in dialysis patients: analysis of surgically excised specimens and review of the literature. Pathol Res Pract. 2016;212:919-26.

10. Ban S, Suzuki S, Kubota K, et al. Gastric mucosal status susceptible to lanthanum deposition in patients treated with dialysis and lanthanum carbonate. Ann Diagn Pathol. 2017;26:6-9.

11. Iwamuro $M$, Sakae $H$, Okada $H$. White gastric mucosa in a dialysis patient. Gastroenterology. 2016;150:322-3.

12. Shitomi Y, Nishida H, Kusaba T, et al. Gastric lanthanosis (lanthanum deposition) in dialysis patients treated with lanthanum carbonate. Pathol Int. 2017;67:389-97.

13. Hoda RS, Sanyal S, Abraham JL, et al. Lanthanum deposition from oral lanthanum carbonate in the upper gastrointestinal tract. Histopathology. 2017;70:1072-8.

14. Iwamuro M, Urata $H$, Tanaka $T$, et al. Lanthanum deposition in the stomach: usefulness of scanning electron microscopy for its detection. Acta Med Okayama. 2017;71:73-8.
15. Kimura M, Kashida H, Enoki H, et al. Early histologic detection of lanthanum deposition in gastric mucosa after lanthanum carbonate therapy. Off J Jpn Soc Lab Med. 2017;65:413-8.

16. Murakami N, Yoshioka M, Iwamuro M, et al. Clinical characteristics of seven patients with lanthanum phosphate deposition in the stomach. Intern Med. 2017;56:2089-95.

17. Iwamuro M, Urata $\mathrm{H}$, Tanaka $\mathrm{T}$, et al. Lanthanum deposition in the stomach in the absence of Helicobacter pylori infection. Intern Med. 2018;57:801-6.

18. Miyamoto S, Kato M, Tsuda M, et al. Gastric mucosal cracked and cobblestone-like changes resulting from proton pump inhibitor use. Dig Endosc. 2017;29:307-13.

19. Takahari K, Haruma K, Ohtani H, et al. Proton pump inhibitor induction of gastric cobblestone-like lesions in the stomach. Intern Med. 2017;6(20):2699-703.

20. Yao K, Iwashita A, Tanabe H, et al. White opaque substance within superficial elevated gastric neoplasia as visualized by magnification endoscopy with narrow-band imaging: a new optical sign for differentiating between adenoma and carcinoma. Gastrointest Endosc. 2008;68:574-80.

21. Yao K, Iwashita A, Nambu M, et al. Nature of white opaque substance in gastric epithelial neoplasia as visualized by magnifying endoscopy with narrow-band imaging. Dig Endosc. 2012;24:419-25.

Publisher's Note Springer Nature remains neutral with regard to jurisdictional claims in published maps and institutional affiliations. 